\title{
Evaluating the impact of intrusion sensitivity on securing collaborative intrusion detection networks against SOOA
}

Madsen, David; Li, Wenjuan; Meng, Weizhi; Wang, Yu

Published in:

Proceedings of $18<$ sup $>$ th $</$ sup $>$ International Conference on Algorithms and Architectures for Parallel Processing

Link to article, DOI:

10.1007/978-3-030-05063-4_36

Publication date:

2018

Document Version

Peer reviewed version

Link back to DTU Orbit

Citation (APA):

Madsen, D., Li, W., Meng, W., \& Wang, Y. (2018). Evaluating the impact of intrussion sensitivity on securing collaborative intrusion detection networks against SOOA. In Proceedings of 18 International Conference on Algorithms and Architectures for Parallel Processing (pp. 481-494). Springer. Lecture Notes in Computer Science (including subseries Lecture Notes in Artificial Intelligence and Lecture Notes in Bioinformatics) Vol. 11337 https://doi.org/10.1007/978-3-030-05063-4_36

\section{General rights}

Copyright and moral rights for the publications made accessible in the public portal are retained by the authors and/or other copyright owners and it is a condition of accessing publications that users recognise and abide by the legal requirements associated with these rights.

- Users may download and print one copy of any publication from the public portal for the purpose of private study or research.

- You may not further distribute the material or use it for any profit-making activity or commercial gain

- You may freely distribute the URL identifying the publication in the public portal 


\title{
Evaluating the Impact of Intrusion Sensitivity on Securing Collaborative Intrusion Detection Networks Against SOOA
}

\author{
David Madsen ${ }^{1}$, Wenjuan $\mathrm{Li}^{1,2}$, Weizhi Meng ${ }^{1 \star}$, and Yu Wang ${ }^{3 \star \star}$ \\ 1 Department of Applied Mathematics and Computer Science, Technical University \\ of Denmark, Denmark \\ 2 Department of Computer Science, City University of Hong Kong, Hong Kong \\ 3 School of Computer Science, Guangzhou University, China \\ weme@dtu.dk; yuwang@gzhu.edu.cn
}

\begin{abstract}
Cyber attacks are greatly expanding in both size and complexity. To handle this issue, research has been focused on collaborative intrusion detection networks (CIDNs), which can improve the detection accuracy of a single IDS by allowing various nodes to communicate with each other. While such collaborative system or network is vulnerable to insider attacks, which can significantly reduce the advantages of a detector. To protect CIDNs against insider attacks, one potential way is to enhance the trust evaluation among IDS nodes, i.e., by emphasizing the impact of expert nodes. In this work, we adopt the notion of intrusion sensitivity that assigns different values of detection capability relating to particular attacks, and evaluate its impact on defending against a special On-Off attack (SOOA). In the evaluation, we investigate the impact of intrusion sensitivity in a simulated CIDN environment, and experimental results demonstrate that the use of intrusion sensitivity can help enhance the security of CIDNs under adversarial scenarios, like SOOA.
\end{abstract}

Keywords: Intrusion Detection, Collaborative Network, Insider Attack, Intrusion Sensitivity, Challenge-based Trust Mechanism.

\section{Introduction}

To help identify and handle various threats, an intrusion detection system (IDS) is often deployed in different security-sensitive environments [31,34]. Generally, there are two types of detection systems according to the deployment: host-based IDS (HIDS) and network-based IDS (NIDS). Each kind of IDS can utilize two detection approaches: signature-based detection and anomaly-based detection. A signature (or rule) is a kind of description on known threat or exploit, in which a signature-based IDS can compare its signatures with incoming events [33,39]. By contrast, anomaly-based detection discovers malicious events by building a

\footnotetext{
^ The author was previously known as Yuxin Meng.
}

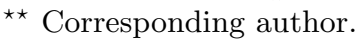


normal profile. An alarm will be notified, if an accurate match is identified or the deviation between the normal profile and current profile exceeds a threshold.

Nowadays, cyber attacks have become much more complicated; thus, a single detector could be easily compromised and ineffective in detecting advanced attacks. To improve the detection performance, collaborative IDS (CIDS) or collaborative intrusion detection network (CIDN) is developed, which allows a set of IDS nodes to communicate with each other and exchange environmental information [40]. In practical setup, a CIDS or CIDN would be vulnerable to insider attacks, where an attacker can perform suspicious actions within a system or a network environment. To address this issue, designing more effective trust evaluation is one promising solution, whereas like challenge-based trust mechanisms, which compute the trustworthiness of a node by sending challenges in a periodic way [6]. However, such trust mechanisms could be compromised by some advanced attacks, i.e., Li et al. [18] developed an advanced collusion attack, a Special On-Off Attack, named SOOA, which can keep giving truthful responses to one node while providing untruthful answers to other nodes.

Contributions. An alternative way of improving the trust evaluation is to emphasize the impact of expert nodes. Li et al. $[13,16]$ identified that different IDS nodes may have different levels of sensitivity in detecting particular intrusions. Then, they introduced a notion of intrusion sensitivity that measures the detection sensitivity of an IDS in detecting different kinds of intrusions. As an example, if a signature-based detector owns more signatures (or rules) in detecting DoS attacks, then it should be more powerful in detecting such specific kind of attack as compared to other nodes, which have relatively fewer signatures. In this work, we attempt to evaluate the impact of intrusion sensitivity on identifying an advanced insider attack, named special On-Off attack (SOOA). The contributions of this work can be summarized as below:

- We first introduce the notion of intrusion sensitivity and explain how to compute trust values of different CIDN nodes. In this work, we focus on a specific kind of advanced insider attack, called special On-Off attack (SOOA), which can maintain the reputation by responding normally to one node while acting abnormally to another node.

- In the evaluation, we investigate the impact of intrusion sensitivity on detecting SOOA in a simulated CIDN environment. Experimental results demonstrate that intrusion sensitivity can be used to improve the security of CIDNs by highlighting the impact of expert nodes in identifying malicious nodes, i.e., it can help decrease the reputation of SOOA nodes faster.

The remaining sections are organized as follows. Section 2 reviews related studies on distributed and collaborative intrusion detection and introduces the background of challenge-based CIDNs. In Section 3, we introduce how SOOA works with two attacking scenarios. Section 4 describes the notion of intrusion sensitivity and evaluates its impact on defending against SOOA in a simulated network environment. Finally, we conclude the work in Section 5. 


\section{Related Work and Background}

This section first introduces related work on intrusion detection, especially collaborative intrusion detection, and then describes the background of challengebased trust mechanism for CIDNs.

\subsection{Related Work}

In a real-world application, a single IDS usually has no information about the protected environment where it is deployed, hence the detector is very easy to be bypassed under some advanced attacks [40]. To address this issue, one effective solution is to construct a distributed or collaborative detection network. Some previously developed distributed systems can be classified as below. 1) Centralized/Hierarchical systems: Emerald [32] and DIDS [35]; 2) Publish/subscribe systems: COSSACK [30] and DOMINO [41]; and 3) P2P Querying-based systems: Netbait [1] and PIER [11].

Generally, collaborative or distributed intrusion detection networks enable an IDS node to achieve more accurate detection by collecting and communicating information from/with other IDS nodes. However, it is well-recognized by the literature that existing collaborative networks are vulnerable to insider attacks. The previous work [12] figured out that most distributed intrusion detection systems (DIDS) relied on centralized fusion, or distributed fusion with unscalable communication mechanisms. Then they gave a solution by designing a distributed detection system based on the decentralized location and routing infrastructure. However, their system is vulnerable to insider attacks, as they assumes that all peers are trusted. Li et al. [18] developed an advanced collusion attack, a Special On-Off Attack, named SOOA, which can keep giving truthful responses to one node while providing untruthful answers to other nodes. They further developed an advanced collusion attack, called passive message fingerprint attack (PMFA) [17], which can compromise the challenge mechanis$\mathrm{m}$ through passively collecting messages and distinguishing normal requests. As such, malicious nodes can maintain their trust values by giving false information to only normal request while providing truthful feedback to other messages.

To protect distributed systems against insider attacks, building appropriate trust models is one of the promising solutions. For instance, Duma et al. [3] proposed a P2P-based overlay IDS to examine traffic by designing a trust-aware engine for handling alerts and an adaptive scheme for managing reputation among different nodes. The former is capable of reducing warnings sent by untrusted or low quality peers, while the latter attempts to predict their trustworthiness by evaluating their past experiences. Tuan [37] then utilized game theory to model and analyze the processes of reporting and exclusion in a $\mathrm{P} 2 \mathrm{P}$ network. They identified that if a reputation system was not incentive compatible, the more numbers of peers in the system, the less likely that anyone will report about a malicious peer. Fung et al. initialized a type of challenge-based CIDNs, in which the reputation level of a node depends mainly on the received answers to the 
challenges. In the beginning, they focus on host-based detection (HIDS) and proposed a host-based collaboration framework that enables each node to evaluate the trustworthiness of others based on its own experience and a forgetting factor [6]. The forgetting factor is used to highlight the recent experience of peers, in order to judge the reputation more effectively.

The concept of intrusion sensitivity was proposed by Li et al. [13], in which they identified that different IDS nodes may have distinct capability or sensitivity in detecting particular types of attacks. Based on the notion, they further developed a trust management model for CIDNs through allocating intrusion sensitivity via machine learning techniques in an automatic way [14]. This concept can help detect intrusions and correlate alarms by emphasizing the impact of an expert IDS. They also studied how to apply intrusion sensitivity for aggregating alarms and defending against pollution attacks, in which a group of malicious peers collaborate together by providing false alarm rankings [15]. Some other related work regarding how to enhance the performance of IDSs can be referred to $[4,5,10,19-29]$

\subsection{Background on Challenge-based CIDNs}

The goal of developing challenge-based trust mechanisms is to help protect CIDNs against insider threats through sending challenges in a periodic manner. Fig. 1 depicts the typical architecture of a challenge-based CIDN. In addition to an IDS module, a CIDN node often contains three major components: trust management component, collaboration component and P2P communication [17].

- Trust management component is responsible for evaluating the reputation of other nodes via a specific trust approach. Challenge-based mechanism is a kind of trust approach that computes the trust values through comparing the received feedback with the expected answers. Each node can send out either normal requests or challenges for alert ranking (consultation). To further protect challenges, the original work [6] assumed that challenges should be sent out in a random manner and in a way that makes them difficult to be distinguished from a normal alarm ranking request.

- Collaboration component is mainly responsible for assisting a node in computing the trust values of another node by sending out normal requests or challenges, and receiving the relevant feedback. This component can help a tested node deliver its feedback when receiving a request or challenge. For instance, Fig. 1 shows that when node $A$ sends a request or challenge to node $B$, it can receive relevant feedback.

- P2P communication. This component is responsible for connecting with other IDS nodes and providing network organization, management and communication among IDS nodes.

Network Interactions. In a CIDN, each node can choose its partners based on its own policies and experience, and maintain a list of collaborated nodes, called 


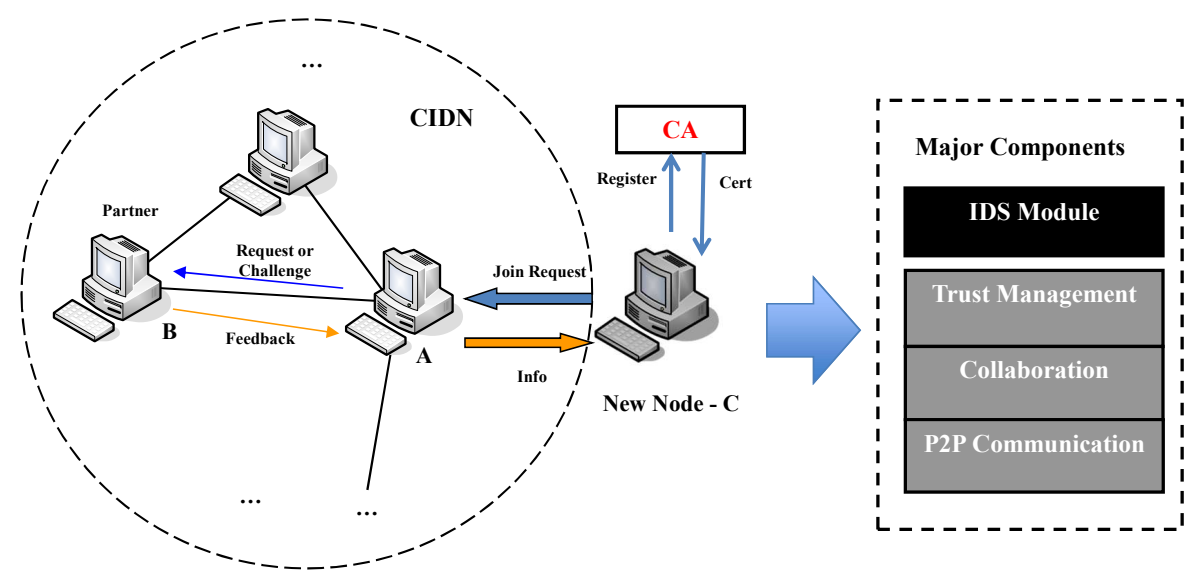

Fig. 1. The typical high-level architecture of a challenge-based CIDN with its major components.

partner list. This list is customizable and stores the relevant information of other nodes like current trust values. Before a node asks for joining the network, A node has to obtain its unique proof of identity (e.g., a public key and a private key) by registering to a trusted certificate authority $(C A)$. As depicted in Fig. 1, if node $C$ asks for joining the network, it has to send a request to a CIDN node, say node $A$. Then, node $A$ makes a decision and sends back an initial partner list, if node $C$ is accepted. A CIDN node can typically send two types of messages: challenge and normal request.

- A challenge mainly contains a set of IDS alarms, where a testing node can send these alarms to the tested nodes for labeling alarm severity. Because the testing node knows the severity of these alarms in advance, it can judge and compute the satisfaction level for the tested node, based on the received feedback.

- A normal request is sent by a node for alarm aggregation, which is an important feature of collaborative networks in improving the detection performance of a single detector. The aggregation process usually only considers the feedback from highly trusted nodes. As a response, an IDS node should send back alarm ranking information as their feedback.

\section{Special On-Off Attack}

Previous work has identified that challenge-based trust mechanism may be still vulnerable to advanced insider attacks, like a special On-Off attack (SOOA) [18], where a malicious node can keep sending truthful responses to one node, but sending malicious responses to another. This attack has a potential to affect the effectiveness of trust computation for a third node (target node). Here, we accept 


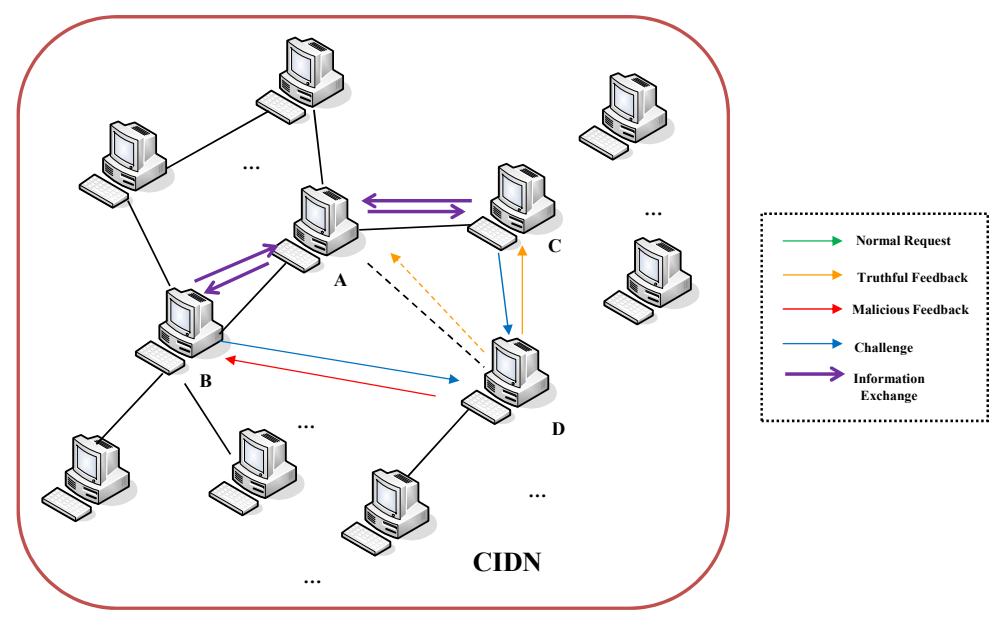

Fig. 2. A special On-Off attack (SOOA) on challenge-based CIDNs.

that a challenge can be sent in a random manner and cannot be distinguished from normal messages in a effective way. Fig. 2 describes an example of SOOA: suppose node $D$ is malicious and node $A$ is the attack target, while node $B$ and node $C$ are two partner nodes for node $A$. Two attacking scenarios can be considered as below [18].

- Scenario 1: node $D$ is not a partner node for node $A$. Under this condition, node $D$ keeps sending truthful response to node $C$ while sending malicious feedback to node $B$. Fig. 2 shows that node $A$ has to communicate and collect data from its partner nodes $B$ and $C$. Subsequently, node $A$ may receive different (or even opposite) reports on node $D$. This scenario often occurs under a hierarchical network structure, in which a central server has to collect information from other nodes and compute the trustworthiness.

- Scenario 2: node $D$ is a partner node for node $A$. Under this condition, node $D$ keeps sending truthful information to node $A$, if they are partner nodes. In a challenge-based CIDNs, node $A$ has to judge the trustworthiness of node $D$ through both its own trust computation and the judgement from other nodes. As a result, this special attack can maintain the reputation of node $D$ over the threshold regarding node $A$.

To summarize, SOOA nodes can keep providing truthful feedback to several nodes, while responding maliciously to others. In this case, it may influence the trust computation of certain nodes and maintain its trust values over the threshold. Malicious nodes thus have a good chance to make a negative impact on alarm aggregation of testing node without decreasing their trust values. In this work, we mainly focus on Scenario 2, since a CIDN node usually aggregates alarms by collecting relevant information from its partner nodes. 


\section{The Impact of Intrusion Sensitivity}

In this section, we first detail the notion of intrusion sensitivity, and then introduce how to setup a CIDN and compute trust values (satisfaction levels).

\subsection{Intrusion Sensitivity}

The previous work [13] found that each IDS should have different sensitivity levels in detecting particular kinds of intrusions and introduced a concept of intrusion sensitivity as below.

- Intrusion sensitivity describes different levels of detection capability (or accuracy) for IDS nodes in detecting particular kinds of attacks or anomalies. Let $I_{s}$ denote the detection sensitivity of a node and $t$ denote a time period. For two IDS nodes $A$ and $B$, we can say $I_{s}^{A}>I_{s}^{B}$ if A has a stronger detection capability within this time period.

Obviously, it is time consuming to assign the values manually in a large network. To automate the allocation of intrusion sensitivity, we can apply machine learning technique. In this work, we adopt a KNN classifier for value allocation based on the following reasons [16]:

- It is easy to implement a KNN algorithm, which classifies objects based on the closest training examples in the feature space. That is, an object can be classified in terms of its distances to the nearest cluster.

- This classifier can also achieve a faster speed with lower computational burden than other classifiers like neural networks in the phases of both training and classification. These properties are desirable when a classifier is deployed in a resource-constrained platform like an IDS node.

It is worth noting that how to objectively allocate the value is still an open challenge, as experts may give different scores for an IDS node, based on their own experience. A potential solutions is to make appropriate specifications and criterion, but it is out of the scope of this paper. To train this classifier, there are generally two steps as follows:

- We first build a classifier model by obtaining the intrusion sensitivity scores for some nodes based on expert knowledge, i.e., some scores given by different security administrators or experts regarding existing nodes.

- When evaluating the intrusion sensitivity of a target node $i$, we use the KNN classifier to assign a value to node $i$ as $I_{s}^{i}$ by running the established model.

\subsection{CIDN Settings}

In this experiment, we constructed a simulated CIDN environment with $50 \mathrm{n}$ odes, which were randomly distributed in a $10 \times 10$ grid region. Each IDS node adopted Snort [36] as IDS plugin. All nodes can communicate with each other 
Table 1. Simulation parameters in the experiment.

\begin{tabular}{|c|c|c|}
\hline Parameters & Value & Description \\
\hline \hline$\lambda$ & 0.9 & Forgetting factor \\
\hline$\varepsilon_{l}$ & $10 /$ day & Low request frequency \\
\hline$\varepsilon_{h}$ & $20 /$ day & High request frequency \\
\hline$r$ & 0.8 & Trust threshold \\
\hline$T_{s}$ & 0.5 & Trust value for newcomers \\
\hline$m$ & 10 & Lower limit of received feedback \\
\hline$d$ & 0.3 & Severity of punishment \\
\hline
\end{tabular}

and build an initial partner list. The trust values of all nodes in the partner list were initialized as $T_{s}=0.5$ based on the results in [6]. According to [16], we set the number of alarms to 40 in either a normal request or a challenge, in order to achieve good classification accuracy.

To evaluate the trustworthiness of partner nodes, each node can send out challenges randomly to its partners with an average rate of $\varepsilon$. There are two levels of request frequency: $\varepsilon_{l}$ and $\varepsilon_{h}$. The request frequency is low for a highly trusted or highly untrusted node, as it should be very confident about their feedback. On the other hand, the request frequency should be high for other nodes whose trust values are close to the threshold. To facilitate comparisons, all the settings can be referred to similar studies $[6,14,18]$. The detailed parameters are shown in Table 1.

Node expertise. This work adopted three expertise levels for an IDS node as: low (0.1), medium (0.5) and high (0.95). A beta function was utilized to model the expertise of an IDS:

$$
\begin{aligned}
& f\left(p^{\prime} \mid \alpha, \beta\right)=\frac{1}{B(\alpha, \beta)} p^{\alpha-1}\left(1-p^{\prime}\right)^{\beta-1} \\
& B(\alpha, \beta)=\int_{0}^{1} t^{\alpha-1}(1-t)^{\beta-1} d t
\end{aligned}
$$

where $p^{\prime}(\in[0,1])$ is the probability of intrusion examined by the IDS. $f\left(p^{\prime} \mid \alpha, \beta\right)$ indicates the probability that a node with expertise level $l$ responses with a value of $p^{\prime}$ to an intrusion examination of difficulty level $d(\in[0,1])$. A higher value of $l$ indicates a higher probability of correctly identifying an intrusion, while a higher value of $d$ indicates that an intrusion is harder to figure out. In particular, $\alpha$ and $\beta$ can be defined as below [7]:

$$
\begin{aligned}
& \alpha=1+\frac{l(1-d)}{d(1-l)} r \\
& \beta=1+\frac{l(1-d)}{d(1-l)}(1-r)
\end{aligned}
$$


where $r \in\{0,1\}$ is the expected detection output. For a fixed difficulty level, the node with a higher level of expertise can achieve a higher probability of correctly identifying an attack. For instance, a node with expertise level of 1 can accurately identify an intrusion with guarantee if the difficulty level is 0 .

Trust evaluation at nodes. To calculate the trust value of a CIDN node, a testing node can send a challenge to the target node via a random generation process, and then compute its satisfaction level by comparing the received feedback with the expected answers. Based on [6], we can evaluate the trustworthiness of a node $i$ according to node $j$ in the following manner:

$$
T_{i}^{j}=\left(w_{s} \frac{\sum_{k=0}^{n} F_{k}^{j, i} \lambda^{t k}}{\sum_{k=0}^{n} \lambda^{t k}}-T_{s}\right)(1-x)^{d} I_{s}^{i}+T_{s}
$$

where $F_{k}^{j, i} \in[0,1]$ is the score of the received feedback $k$ and $n$ is the total number of feedback. $\lambda$ is a forgetting factor that assigns less weight to older feedback. $w_{s}$ is a significant weight relying on the total number of received feedback, if there is only a few received feedback under a certain minimum $m$, then $w_{s}=\frac{\sum_{k=0}^{n} \lambda^{t k}}{m}$; otherwise $w_{s}=1 . x$ is the percentage of "don't know" answers for a period of time (e.g., from $t 0$ to $t n$ ). $d$ is a positive incentive parameter to control the severity of punishment to "don't know" answers. $I_{s}^{i}(\in[0,1])$ is the intrusion sensitivity of node $i$.

Satisfaction evaluation. Suppose there are two factors: an expected feedback $(e \in[0,1])$ and an actual received feedback $(r \in[0,1])$. Then, this work used a function $F(\in[0,1])$ to reflect the satisfaction level by measuring the difference between the received answer and the expected answer as below [7].

$$
\begin{array}{ll}
F=1-\left(\frac{e-r}{\max \left(c_{1} e, 1-e\right)}\right)^{c_{2}} & e>r \\
F=1-\left(\frac{c_{1}(r-e)}{\max \left(c_{1} e, 1-e\right)}\right)^{c_{2}} & e \leq r
\end{array}
$$

where $c_{1}$ controls the degree of penalty for wrong estimates and $c_{2}$ controls satisfaction sensitivity. Based on the work [7], we set $c_{1}=1.5$ and $c_{2}=1$.

\subsection{Experimental Results}

In this experiment, we aim to evaluate the impact of intrusion sensitivity on the security of CIDNs. Fig. 3 illustrates the convergence of trust values regarding different expert nodes with three expertise levels: low $(I=0.1)$, medium $(I=0.5)$ and high $(I=0.95)$. It is found that the nodes with higher expertise can achieve higher reputation levels. In this simulated environment, all nodes' reputation levels turned stable after around 20 days, since challenge-based trust mechanism requires a long time for establishing a high trust value. 


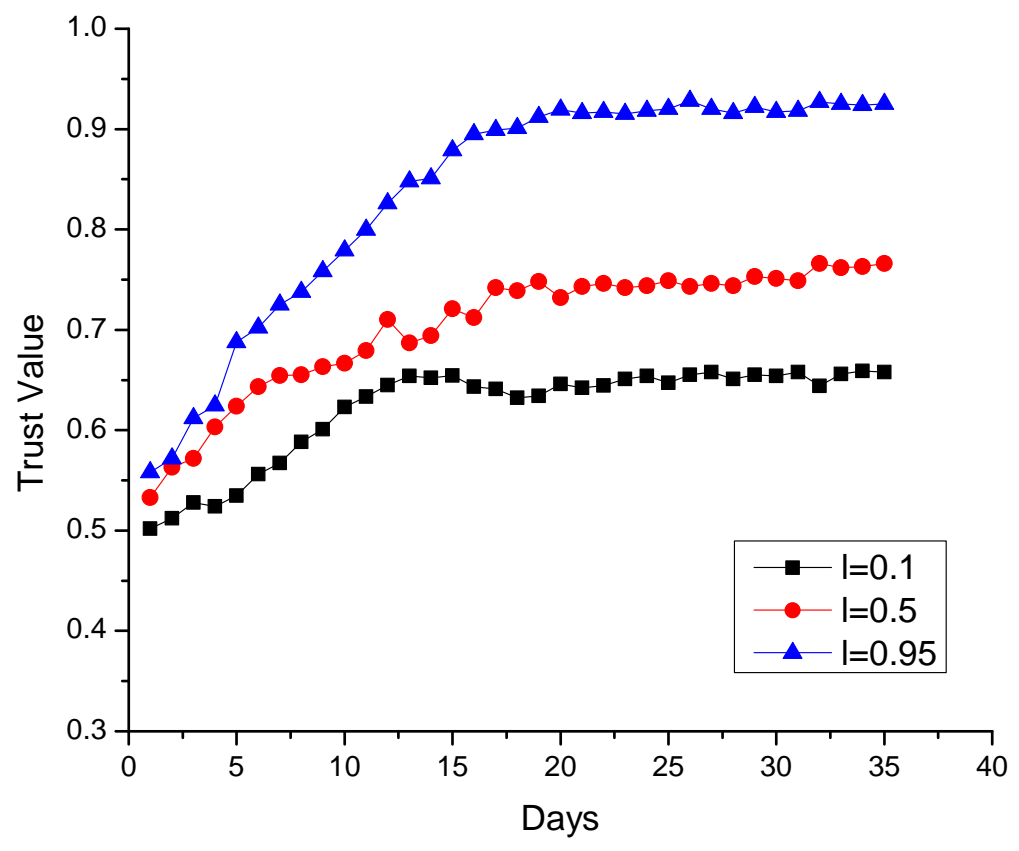

Fig. 3. Convergence of trust values of IDS nodes regarding three expertise levels.

The impact of SOOA. According to Fig. 2, we suppose node $A$ has seven partner nodes and node $D$ is a partner node for node $A$. Similar to [18], we assume that node $D$ keeps sending truthful feedback to several partner nodes of node $A$, while sending untruthful answers to the rest partner nodes. We randomly selected one expert node $(I=0.95)$ as malicious (say node $D$ ), which conducted the special attack of SOOA from Day 45. For a scenario of $4 \mathrm{~T} 2 \mathrm{U}$, node $D$ can send truthful feedback to four partner nodes of node $A$ but sent untruthful feedback to the remaining two partner nodes. Fig. 4 depicts the trust value of node $D$ under this condition. It is found that the trust value of node $D$ computed by node $A$ could gradually decrease closer to the threshold during the first ten days, because two partner nodes could report malicious actions regarding node $D$ to node $A$. Afterwards, the trust value was maintained in the range from 0.82 to 0.83 at most cases, as there are still four partner nodes reported that node $D$ is normal. As the trust value is higher than the threshold 0.8 , node $D$ still has an impact on node $A$ and its alarm aggregation.

The impact of intrusion sensitivity. In the same CIDN environment, we assume that under $4 \mathrm{~T} 2 \mathrm{U}$, node $D$ keeps sending untruthful feedback to two partner nodes, which are expert nodes. Fig. 4 shows the impact of intrusion sensitivity on the detection of malicious SOOA nodes. It is found that the reputation levels of malicious node steadily decreased below the threshold of 0.8 (i.e., within several days). This is because the use of intrusion sensitivity can give more weights 


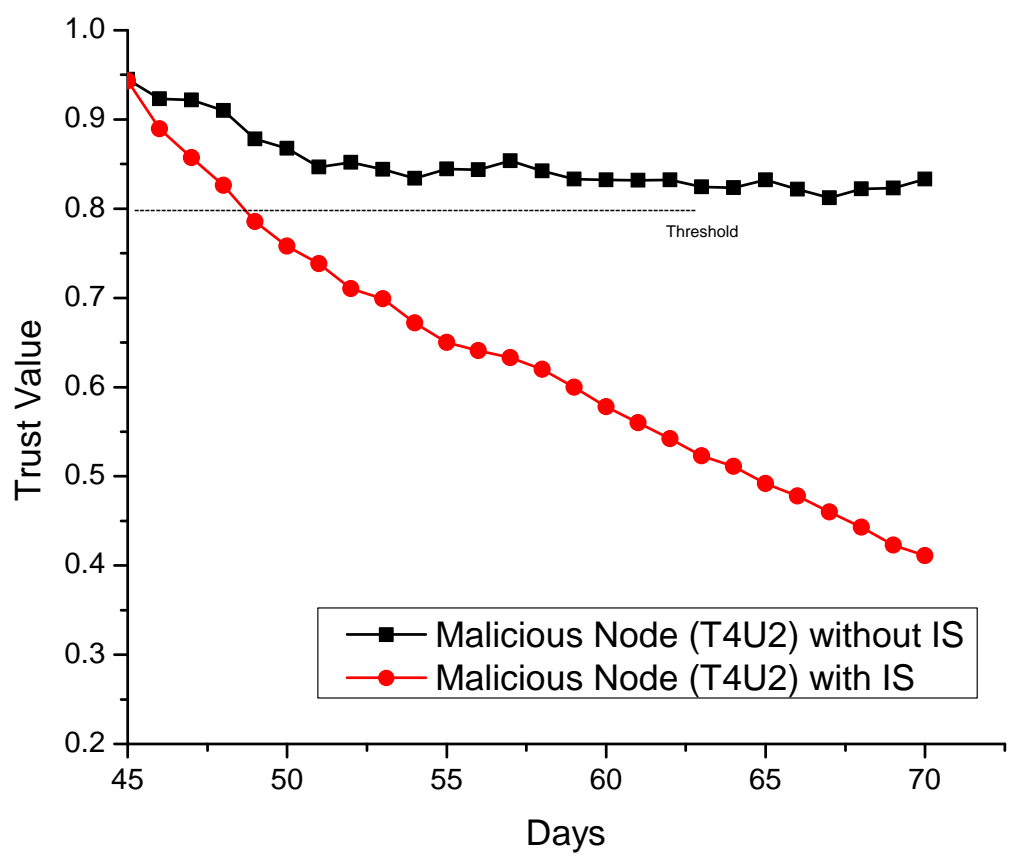

Fig. 4. Trust values of malicious nodes with and without intrusion sensitivity (IS).

on the feedback from expert nodes. The result demonstrated that this notion could help improve the robustness of CIDNs by reducing the reputation levels of malicious nodes under SOOA in a fast manner.

\subsection{Discussion}

In this work, we investigate the impact of intrusion sensitivity on securing CIDNs against SOOA, whereas some challenges still remain for future work.

- Additional measurement. In challenge-based CIDNs, the trustworthiness of a node is mainly determined by challenges, whereas it may still leave a chance for attackers. To further increase the robustness of CIDNs, additional measures can be used to calculate the trust values of a node, like packet-level trust [26].

- Scalability. In this work, we explored the influence under a simulated environment, but we did not perform a particular experiment to investigate the scalability issue. This is an interesting topic for our future work.

- Advanced insider attacks. In this work, we mainly focus on SOOA, an advanced collusion attack for challenge-based CIDNs. In the literature, there are many kinds of advanced insider attacks like FPMA [17]. It is an important topic to examine the influence on other attacks. 


\section{Conclusion}

As a CIDS or CIDN allows a set of IDS nodes to communicate with each other, it can enhance the detection performance of a single detector. However, such system or network is vulnerable to insider attacks, where an attacker can behave maliciously within a system or a network environment. In this work, we adopt the notion of intrusion sensitivity that assigns different values of detection capability relating to particular attacks, and evaluate its impact on securing CIDNs against a special insider attack, SOOA. In the evaluation, we investigate the impact of intrusion sensitivity in a simulated CIDN environment, and the obtained results demonstrate that intrusion sensitivity can help enhance the security of CIDNs under adversarial scenarios, through emphasizing the input from expert nodes. Our work attempts to stimulate more research in designing more secure CIDNs in real-world scenarios. Future work could include exploring the impact of intrusion sensitivity on defending other insider attacks like PMFA.

\section{References}

1. Chun, B., Lee, J., Weatherspoon, H., Chun, B.N.: Netbait: a Distributed Worm Detection Service. Technical Report IRB-TR-03-033, Intel Research Berkeley (2003)

2. Douceur, J.: The sybil attack. In: Druschel, P., Kaashoek, M.F., Rowstron, A. (eds.) IPTPS 2002. LNCS, vol. 2429. Springer, Heidelberg (2002)

3. Duma, C., Karresand, M., Shahmehri, N., Caronni, G.: A Trust-Aware, P2P-Based Overlay for Intrusion Detection. In: DEXA Workshop, pp. 692-697 (2006)

4. Fadlullah, Z.M.,Taleb, T.,Vasilakos, A.V.,Guizani, M.,Kato, N.: DTRAB: combating against attacks on encrypted protocols through traffic-feature analysis. IEEE/ACM Transactions on Networking, vol. 18, no. 4, pp. 1234-1247 (2010)

5. Friedberg, I., Skopik, F., Settanni, G., Fiedler, R.: Combating advanced persistent threats: From network event correlation to incident detection. Computers \& Security, vol 48, pp. 35-47 (2015)

6. Fung, C.J., Baysal, O., Zhang, J., Aib, I., Boutaba, R.: Trust Management for Host-Based Collaborative Intrusion Detection. In: De Turck, F., Kellerer, W. Kormentzas, G. (eds.): DSOM 2008, LNCS 5273, pp. 109-122 (2008)

7. Fung, C.J., Zhang. J., Aib, I., Boutaba, R.: Robust and scalable trust management for collaborative intrusion detection. In: Proceedings of the 11th IFIP/IEEE International Conference on Symposium on Integrated Network Management (IM), pp. 33-40 (2009)

8. Ghosh, A.K., Wanken, J., Charron, F.: Detecting Anomalous and Unknown Intrusions Against Programs. In: Proceedings of Annual Computer Security Applications Conference (ACSAC), pp. 259-267 (1998)

9. Gong, F.: Next Generation Intrusion Detection Systems (IDS). McAfee Network Security Technologies Group (2003)

10. Gou, Z., Ahmadon, M.A.B., Yamaguchi, S., Gupta, B.B.: A Petri Net-based Framework of Intrusion Detection Systems. In: Proceedings of the 4th IEEE Global Conference on Consumer Electronics, pp. 579-583 (2015) 
11. Huebsch, R., Chun, B.N., Hellerstein, J.M., Loo, B.T., Maniatis, P., Roscoe, T., Shenker, S., Stoica, I., Yumerefendi, A.R.: The Architecture of PIER: an InternetScale Query Processor. In: Proceedings of the 2005 Conference on Innovative Data Systems Research (CIDR), pp. 28-43 (2005)

12. Li, Z., Chen, Y., Beach, A.: Towards Scalable and Robust Distributed Intrusion Alert Fusion with Good Load Balancing. In: Proceedings of the 2006 SIGCOMM Workshop on Large-Scale Attack Defense (LSAD), pp. 115-122 (2006)

13. Li, W., Meng, Y., Kwok, L.-F.: Enhancing Trust Evaluation Using Intrusion Sensitivity in Collaborative Intrusion Detection Networks: Feasibility and Challenges. In: Proceedings of the 9th International Conference on Computational Intelligence and Security (CIS), pp. 518-522, IEEE (2013)

14. Li, W., Meng, Y., Kwok, L.-F.: Design of Intrusion Sensitivity-Based Trust Management Model for Collaborative Intrusion Detection Networks. In: Proceedings of the 8th IFIP WG 11.11 International Conference on Trust Management (IFIPTM), Springer, pp. 61-76 (2014)

15. Li. W., Meng, W.: Enhancing Collaborative Intrusion Detection Networks Using Intrusion Sensitivity in Detecting Pollution Attacks. Information and Computer Security 24(3), pp. 265-276, Emerald (2016)

16. Li, W., Meng, W., Kwok, L.-F., Ip, H.H.S.: Enhancing collaborative intrusion detection networks against insider attacks using supervised intrusion sensitivitybased trust management model. Journal of Network and Computer Applications 77, pp. 135-145 (2017)

17. Li, W., Meng, W., Kwok, L.F., Ip, H.H.S.: PMFA: Toward Passive Message Fingerprint Attacks on Challenge-based Collaborative Intrusion Detection Networks. In: Proceedings of the 10th International Conference on Network and System Security (NSS 2016), pp. 433-449 (2016)

18. Li, W., Meng, W., Kwok, L.F.: SOOA: Exploring Special On-Off Attacks on Challenge-Based Collaborative Intrusion Detection Networks. In: Proceedings of GPC, pp. 402-415 (2017)

19. Meng, Y., Kwok, L.F.: Enhancing False Alarm Reduction Using Voted Ensemble Selection in Intrusion Detection. International Journal of Computational Intelligence Systems, vol. 6, no. 4, pp. 626-638, Taylor \& Francis (2013)

20. Meng, Y., Li, W., Kwok, L.F.: Towards Adaptive Character Frequency-based Exclusive Signature Matching Scheme and its Applications in Distributed Intrusion Detection. Computer Networks, vol. 57, no. 17, pp. 3630-3640, Elsevier (2013)

21. Meng, W., Li, W., Kwok, L.-F.: An Evaluation of Single Character FrequencyBased Exclusive Signature Matching in Distinct IDS Environments. In: Proceedings of the 17th International Conference on Information Security (ISC), pp. 465$476(2014)$

22. Meng, W., Li, W., Kwok, L.-F.: EFM: Enhancing the Performance of Signaturebased Network Intrusion Detection Systems Using Enhanced Filter Mechanism. Computers \& Security, vol. 43, pp. 189-204, Elsevier (2014)

23. Meng, W., Li, W., Kwok, L.-F.: Design of Intelligent KNN-based Alarm Filter Using Knowledge-based Alert Verification in Intrusion Detection. Security and Communication Networks 8(18), pp. 3883-3895, Wiley (2015)

24. Meng, W., Au, M.H.: Towards Statistical Trust Computation for Medical Smartphone Networks Based on Behavioral Profiling. In: Proceedings of the 11th IFIP WG 11.11 International Conference on Trust Management (IFIPTM), pp. 152-159 (2017) 
25. Meng, W., Li, W., Xiang, Y., Choo, K.K.R.: A Bayesian Inference-based Detection Mechanism to Defend Medical Smartphone Networks Against Insider Attacks. Journal of Network and Computer Applications, vol. 78, pp. 162-169, Elsevier (2017)

26. Meng, W., Li, W., Kwok, L.-F.: Towards Effective Trust-based Packet Filtering in Collaborative Network Environments. IEEE Transactions on Network and Service Management, vol. 14, no. 1, pp. 233-245 (2017)

27. Meng, W., Wang, Y., Li, W., Liu, Z., Li, J., Probst, C.W.: Enhancing Intelligent Alarm Reduction for Distributed Intrusion Detection Systems via Edge Computing. In: Proceedings of The 23rd Australasian Conference on Information Security and Privacy (ACISP) (2018)

28. Meng, W., Li, W., Wang, Y., Au, M.H.: Detecting Insider Attacks in Medical Cyber-Physical Networks based on Behavioral Profiling. Future Generation Computer Systems, In Press, Elsevier (2018)

29. Mishra, A., Gupta, B.B., Joshi, R.C.: A Comparative study of Distributed Denial of Service Attacks, Intrusion Tolerance and mitigation Techniques. In: Proceedings of the 2011 European Intelligence and Security Informatics Conference, pp. 286-289 (2011)

30. Papadopoulos, C., Lindell, R., Mehringer, J., Hussain, A., Govindan, R.: COSSACK: Coordinated Suppression of Simultaneous Attacks. In: Proceedings of the 2003 DARPA Information Survivability Conference and Exposition (DISCEX), pp. 94-96 (2003)

31. Paxson, V.: Bro: A System for Detecting Network Intruders in Real-Time. Computer Networks, vol. 31, no. 23-24, pp. 2435-2463 (1999)

32. Porras, P.A., Neumann, P.G.: Emerald: Event Monitoring Enabling Responses to Anomalous Live Disturbances. In: Proceedings of the 20th National Information Systems Security Conference, pp. 353-365 (1997)

33. Roesch, M.: Snort: Lightweight intrusion detection for networks. In: Proceedings of Usenix Lisa Conference, pp. 229-238 (1999)

34. Scarfone, K., Mell, P.: Guide to Intrusion Detection and Prevention Systems (IDPS). NIST Special Publication 800-94 (2007)

35. Snapp, S.R., et al.: DIDS (Distributed Intrusion Detection System) - Motivation, Architecture, and An Early Prototype. In: Proceedings of the 14th National Computer Security Conference, pp. 167-176 (1991)

36. Snort: An an open source network intrusion prevention and detection system (IDS/IPS). Homepage: http://www.snort.org/

37. Tuan, T.A.: A Game-Theoretic Analysis of Trust Management in P2P Systems. In: Proceedings of ICCE, pp. 130-134 (2006)

38. Valdes, A., Anderson, D.: Statistical Methods for Computer Usage Anomaly Detection Using NIDES. Technical Report, SRI International (January 1995)

39. Vigna, G.,Kemmerer, R.A.: NetSTAT: A Network-based Intrusion Detection Approach. In: Proceedings of Annual Computer Security Applications Conference (ACSAC), pp. 25-34 (1998)

40. Wu, Y.-S., Foo, B., Mei, Y., Bagchi, S.: Collaborative Intrusion Detection System (CIDS): A Framework for Accurate and Efficient IDS. In: Proceedings of the 2003 Annual Computer Security Applications Conference (ACSAC), pp. 234-244 (2003)

41. Yegneswaran, V., Barford, P., Jha, S.: Global Intrusion Detection in the DOMINO Overlay System. In: Proceedings of the 2004 Network and Distributed System Security Symposium (NDSS), pp. 1-17, (2004) 